\title{
Characterization of Multiwalled Carbon Nanotubes Dispersing in Water and Association with Biological Effects
}

\author{
Xuelian Cheng, ${ }^{1}$ Jun Zhong, ${ }^{2}$ Jie Meng, ${ }^{1}$ Man Yang, ${ }^{1}$ Fumin Jia, ${ }^{1}$ Zhen Xu, ${ }^{1}$ \\ Hua Kong, ${ }^{1}$ and Haiyan $\mathrm{Xu}^{1}$ \\ ${ }^{1}$ Institute of Basic Medical Sciences, Chinese Academy of Medical Sciences and Peking Union Medical College, \\ Beijing 100005, China \\ ${ }^{2}$ Institute of Functional Nano \& Soft Materials, Soochow University, Jiangsu 215123, China
}

Correspondence should be addressed to Haiyan Xu, xuhy@pumc.edu.cn

Received 24 May 2011; Accepted 23 June 2011

Academic Editor: Xing J. Liang

Copyright () 2011 Xuelian Cheng et al. This is an open access article distributed under the Creative Commons Attribution License, which permits unrestricted use, distribution, and reproduction in any medium, provided the original work is properly cited.

\begin{abstract}
Biomedical application potentials of carbon nanotubes-based materials have been investigated intensively in recent years; however, characterization and metrology are still facing great technical challenges when the materials are intended to be used as carriers for therapeutics in aqueous solutions. Systematic characterization on the dispersing carbon nanotubes is urgently required and therefore of significance. In this paper multiwalled carbon nanotubes (MWCNTs) with different average lengths or with different oxidation degrees were dispersed in water and characterized systematically by applying UV spectroscopy, SEM, DLS, TGA, XPS, and FTIR. In particular, the characteristic absorption of the carbon nanotubes was analyzed using resolution-fitting technique to establish relations of wavelength and absorption intensity to the size distribution and surface chemistry. Results indicated that the absorption spectra of MWCNTs could reflect the variation of surface chemistry and length distribution of carbon nanotubes dispersed in water by combining with the other measurements. A vascular endothelium cell line was taken as a model to figure out association between physicochemical features and cytotoxicity of the carbon nanotubes. It was showed that the multiwalled carbon nanotubes with different oxidation degrees and similar length distribution exhibited different interaction files to the cells proliferation in a manner of time dependence and concentration dependence.
\end{abstract}

\section{Introduction}

Carbon nanotubes have shown their promising potentials in biomedical fields including novel delivery systems for drugs or DNAs/RNAs in recent years, which have been reviewed in detail in some publications [1-6]. Meanwhile, biological safety and risks along with the application of carbon nanotubes-based materials have been seriously concerned, as related research publications are increasing constantly and the experimental data from different research groups are often different and even conflicted each other [7-12]. For example, Takagi et al. reported an incidence of mesothelioma in p53-deficient mice injected intraperitoneally with $3 \mathrm{mg}$ per mouse of multiwalled carbon nanotubes [8]. On the contrary, Muller et al. reported that, several months after the injection of nanotubes, the inflammatory reaction was almost absent and limited by a fibrotic encapsulation; hence, multiwalled carbon nanotubes (MWCNTs) with or without structural defects did not induce mesothelioma in this bioassay displaying the absence of carcinogenicity of nanotubes [9]. Accumulating evidence implied that one of the important reasons that cause these conflicts is the lack of standard metrology for carbon nanotubes due to the lack of comprehensive characterization, which makes it difficult to compare data from different laboratories worldwide. Besides making comparison, the great efforts to apply carbon nanotubes into biomedical fields are requiring comprehensive characterization urgently.

For molecular drugs, their physicochemical properties such as molecular weight, chemical composition, purity, solubility, and stability are usually necessary to analyze. The instrumentation to ascertain these properties have been well established, and the techniques are standardized. Techniques 
such as nuclear magnetic resonance (NMR), mass spectrometry, ultraviolet-visible (UV-Vis) spectroscopy, infrared spectroscopy (IR), and gas chromatography (GC) can well meet the demands to analyze such molecules. However, for carbon nanotubes dispersing in water, there are big technical challenges in metrology as well as in characterization.

As it is well known, carbon nanotubes are one representative nanomaterial with heterostructure. The molecular weight of the carbon nanotubes is hard to determine due to their complicated surface chemistry induced by different modification processes and broad length distribution. Besides, they are usually required to disperse in water when being considered to be applied as carriers for therapeutic or detective molecules, while characterizations for carbon nanotubes dispersing in the aqueous solutions is facing more difficulties because most of the existing measurement technologies are just applicable to solid nanomaterials.

It has been noticed that the physicochemical features are likely to affect biological effects of the carbon nanotubes. For instance, some investigations have indicated that size distribution and surface chemistry of carbon nanotubes affected their interactions to the cells. Sato et al. reported that the degree of inflammatory response in subcutaneous tissue in rats induced by the MWCNTs of about $220 \mu \mathrm{m}$ in length was slight in comparison with that around those induced by the MWCNTs of about $825 \mu \mathrm{m}$ in length [13]; Li et al. modified MWCNTs with phosphatidylcholine (PC), polyethylene glycol (PEG), and PC-terminated polyethylene glycol (PEG-PC), and the modified MWCNT induced only low acute toxicity in reference to the original MWCNT [14]. Nevertheless, lots of experimental data are hard to be compared because in many cases only a broad size range or an average length value was given in the literature, and most of them were the description for the carbon nanotubes in the solid phase. These strongly suggest that comprehensive characterization for carbon nanotubes dispersing in the aqueous solution and the association with biological effects still requires extensive investigation.

This work aimed to make systematic and detailed characterization of as-received or oxidized multiwalled carbon nanotubes (MWCNTs) dispersing in water by applying UV-vis-NIR spectroscopy, scanning electron microscope (SEM), dynamic light scattering (DLS), thermogravimetry analysis (TGA), X-ray photoelectron spectroscopy (XPS), and Fourier transform infrared spectroscopy (FTIR). In particular, a resolution-fitting technique was applied with the UV-vis-NIR spectra of the carbon nanotubes to establish relations of wavelength and absorption intensity to the size distribution and surface chemistry. Additionally, a vascular endothelium cell line was taken as a model to figure out association between physicochemical features and cytotoxicity of the carbon nanotubes.

\section{Materials and Methods}

2.1. Materials. Three kinds of as-received multiwalled carbon nanotubes (MWCNTs) were purchased from Chengdu Organic Chemicals Co. Ltd. the diameter for the samples is $20 \sim 30 \mathrm{~nm}$, and average length of the samples is given by the manufacturer as $0.5 \sim 2 \mu \mathrm{m}$ (s-MWCNTs), $30 \mu \mathrm{m}$ (mMWCNTs), and $50 \mu \mathrm{m}$ (l-MWCNTs). The samples purity is $>95 \%$, amorphous carbon $<3 \%$, and ash (catalyst residue) $<1.5 \%$.

2.2. Oxidation of l-MWCNTs. In this work, only as-received MWCNTs were treated to obtain oxidized 1-MWCNTs with different oxidation degrees. The treatment procedure included a combination of concentrated acids oxidation and sonication as described in previous literature [15]. In brief, the as-received l-MWCNTs were mixed in concentrated $\mathrm{H}_{2} \mathrm{SO}_{4} / \mathrm{HNO}_{3}$ ( $3: 1$ by volume) for $12 \mathrm{~h}$, followed by a probe sonication at $750 \mathrm{~W}$ for different times of $0,30,60$, and 100 seconds to make different oxidation degrees; the resulting products are oxidized 1-MWCNTs and named l-MWCNTs- ${ }_{1}, 1-M W C N T s-\mathrm{O}_{2}, 1-M W C N T s-$ $\mathrm{O}_{3}$, and l-MWCNTs- $\mathrm{O}_{4}$, respectively. The above oxidized products were rinsed, filtrated using millipore membrane (pore size: $2 \mu \mathrm{m}$ ) thoroughly with distilled water $(18.2 \Omega$ ) till the $\mathrm{pH}$ value of the running water reached to that of original, and then dried completely in a vacuum oven at $50^{\circ} \mathrm{C}$.

\subsection{UV Spectroscopy Analysis and Peak Resolution and Fitting}

\subsubsection{Preparation of Colloid Solutions of Carbon Nanotubes.} The as-received 1-MWCNTs, m-MWCNTs, and 1-MWCNTs and the oxidized l-MWCNTs samples were dispersed in distilled water or in complete culture medium (that includes DMEM and 10\% FCS) by aid of probe sonication at $360 \mathrm{~W}$ for 60 seconds, followed by a centrifugation of $1540 \mathrm{~g} / \mathrm{min}$ for 20 minutes to remove undispersed substance from the aqueous phase. The solutions were subjected to UV-Vis-NIR spectroscopy (Lambda 950, Perkin-Elmer). The absorption spectra of the samples were resolved into 3 subpeaks and fitted to envelop using software of Igor pro 6.1.

2.3.2. Measurement of Colloids Stability of Carbon Nanotubes. To measure the colloid stability of carbon nanotubes in the water at static condition, the solution samples obtained in Section 2.3.1 were placed in vertically standing tubes and stored at room temperature for $2,8,17,24$, and 31 days. At each time point, a $50 \mu \mathrm{L}$ of the stock solutions of very upper part was taken and subjected to UV-Vis-NIR spectroscopy (Lambda 950, Perkin-Elmer). The optical density (O.D.) of the solutions was measured. For measurements in dynamic condition, the solutions obtained in Section 2.3.1 and stored post 8 days were centrifuged in $4310 \mathrm{~g} / \mathrm{min}, 6740 \mathrm{~g} / \mathrm{min}$, and $11390 \mathrm{~g} / \mathrm{min}$, respectively. The O.D. of the supernatants was measured.

2.4. Scanning Electron Microscopy (SEM). Solutions obtained in Section 2.3.1 were dropped on a silicon substrate and dried at room temperature for scanning electron microscopy (SEM, Hitachi S-5200) observation. Length distribution for the four oxidized MWCNTs was obtained by counting more than 300 nanotubes randomly taken in ten SEM images. 
2.5. Dynamic Light Scattering Measurement. Solutions obtained in Section 2.3.1 were subjected to dynamic light scattering spectroscopy (ZEN 3690; Malvern Instruments Ltd, Malvern, UK) at a fixed scattering angle of 90 at $25^{\circ} \mathrm{C}$. Zeta potential and relative hydrodynamic diameter distribution of the MWCNTs solutions (water or the culture medium) were measured.

2.6. Thermogravimetric Analysis. Samples of as-received and oxidized MWCNTs were weighted and mounted to thermogravimetric analysis (TG209C, NETZSCH). The measurement temperature ranged from $25^{\circ} \mathrm{C}$ to $800^{\circ} \mathrm{C}$, and heating rate is $20^{\circ} \mathrm{C} / \mathrm{min}$.

\subsection{Surface Chemistry Analysis}

2.7.1. FT-IR Analysis. Samples of MWCNTs were analyzed by Fourier transform infrared spectroscopy (FT-IR, Nicolet NEXUS 670). Spectra were recorded with a resolution of $2 \mathrm{~cm}^{-1}$ over the wave number range $4000 \sim 400 \mathrm{~cm}^{-1}$.

2.7.2. XPS Analysis. X-ray photoelectron spectroscopy (XPS) measurements were performed on a Japan JEOL Scientific JPS-9010TR XPS system with Al K Alpha radiation as the exciting source, where the binding energies were calibrated by referencing the $\mathrm{C} 1 \mathrm{~s}$ peak to reduce the sample charge effect.

2.8. MTS Assay to Examine Endothelium Cells Viability. Endothelial cell line (EA.hy926) was purchased from the Cell Bank of Shanghai Institutes of Biological Sciences, Chinese Academy of Sciences (Shanghai, China), which was maintained in DMEM media supplemented with $10 \%$ fetal calf serum, $4 \mathrm{mM}$ L-glutamine, $1 \mathrm{mM}$ sodium pyruvate, $4500 \mathrm{mg} / \mathrm{L}$ glucose, $1500 \mathrm{mg} / \mathrm{L}$ sodium bicarbonate, and $0.1 \%$ penicillin $\mathrm{G}$ and streptomycin (Invitrogen) at $37^{\circ} \mathrm{C}$ with $5 \% \mathrm{CO}_{2}$. Endothelial cells were detached from the culture flask using $0.125 \mathrm{M}$ trypsin-EDTA when they became $70 \sim 80 \%$ confluent. Then the cells were seeded on 96-well plate at a density of $4 \times 10^{3}$ cells/well and cultivated in an incubator overnight. The culture medium was replaced with $200 \mu \mathrm{L}$ of culture medium containing l-MWCNTs$\mathrm{O}_{1}, 1$-MWCNTs- $\mathrm{O}_{2}, 1-\mathrm{MWCNTs}^{-} \mathrm{O}_{3}$, or l-MWCNTs- $\mathrm{O}_{4}$ with concentrations of $0.01,0.05$ and $0.25 \mathrm{mg} / \mathrm{mL}$. The viable cells number was determined at $48 \mathrm{~h}$ and $72 \mathrm{~h}$ using MTS assay (CellTiter 96 @ A Queous Non-Radioactive Cell Proliferation Assay, Promega) according to the manufacturer's instruction. The intact culture medium was taken as control.

The viability of the cells was calculated using the following equation:

Viability $(\%)=\left[\frac{(\text { average cell number of sample wells })}{(\text { average cell number of control wells })}\right]$ $\times 100 \%$.
The data were expressed as average \pm standard deviation $(\bar{x} \pm \mathrm{SD})$ unless otherwise stated. Data were analyzed using a Student's two-tailed test, assuming equal variance with SAS 8.2 software.

\section{Results and Discussion}

3.1. SEM Observation of As-Received MWCNTs and Oxidized MWCNTs. Length distribution for carbon nanotubes dispersing in water is one very crucial parameter because, to a large extent, it determines biological effects of carbon nanotubes in water. Detailed information of length distribution is necessary and helpful to deeply understand biological effects induced by carbon nanotubes.

SEM is widely used to characterize morphology as well as individual size of carbon nanotubes materials [16-20]. As seen in the SEM images shown in Figure 1, the length distribution for as-received s-, m-, and l-MWCNTs which are easy to distinguish is obviously different. In addition, it could be noticed that the tube surface of as-received s- or mMWCNTs seemed not as clear as the surface of as-received lMWCNTs. These indicated other forms of carbon substance on the tubes surface.

Figure 2 presented SEM images of 1-MWCNTs treated with different oxidation degrees as an example of oxidation effect on the length of carbon nanotubes. It was clearly seen that, after oxidation treatment, the typical tube-like structures were remained while the length of as-received 1MWCNTs became obviously shorter in reference to that of original. By statistically counting from randomly selected SEM images, the length distribution of the four oxidized l-MWCNTs was very close, the four samples seemed to have similar size distributions ranging from $500 \mathrm{~nm}$ to $2 \mu \mathrm{m}$, and the average length is $940,967,967$, and $974 \mathrm{~nm}$ for l-MWCNTs- $\mathrm{O}_{1}, 1-\mathrm{MWCNTs}-\mathrm{O}_{2}, 1-\mathrm{MWCNTs}_{3} \mathrm{O}_{3}$, and l-MWCNTs- ${ }_{4}$, respectively (inserted plots in the SEM images).

3.2. DLS Analysis of the Size Distribution of Oxidized MWCNTs. Dynamic light spectroscopy (DLS) is one measurement that may provide information of nanomaterials size distribution. Although DLS measurement is suitable to determine the diameter of particles with sphere shape, it can still provide hydrodynamic diameter for nanotubes $[21,22]$; the data could be used to evaluate variation of length distribution for carbon nanotubes. The measurement of DLS and Zeta potential was conducted with the four kinds of oxidized 1-MWCNTs dispersing in water or in the culture medium.

Zeta potential measurement showed that the four oxidized 1-MWCNTs had very similar Zeta potential values around $-42 \mathrm{mV}$ when dispersing in water (Figure 3(a)), which is much lower than that of as-received l-MWCNTs of $-28 \mathrm{mV}$. It is indicative that the oxidation treatment increased hydrophilicity and dispersion stability of $1-$ MWCNTs in water. When dispersing in the culture medium, Zeta potential of the four kinds of oxidized l-MWCNTs was around $-16 \mathrm{mV}$ (Figure $3(\mathrm{c})$ ), which is very similar to that 


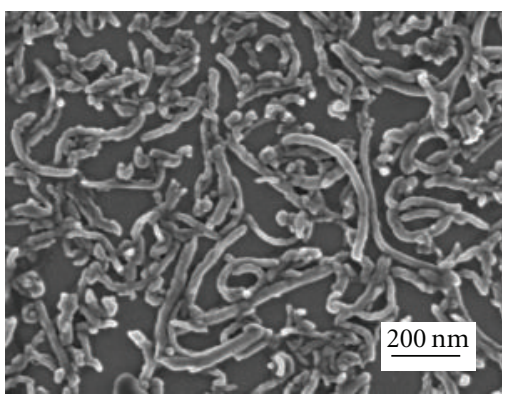

(a)

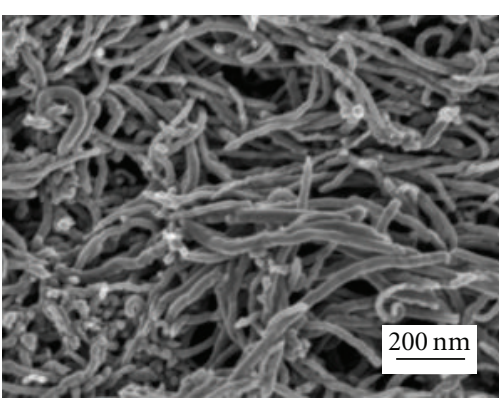

(b)

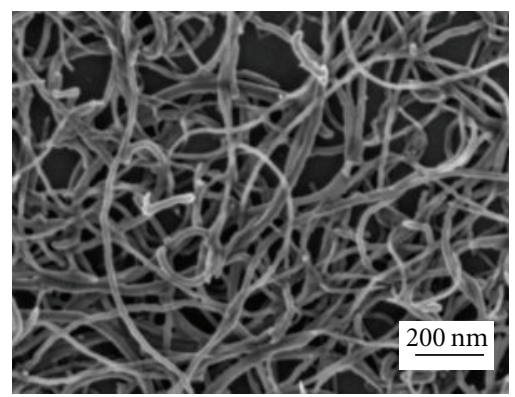

(c)

FIGURE 1: SEM images of three kinds of as-received MWCNTs with different average length: (a) $0.5 \sim 2 \mu \mathrm{m}$, (b) $30 \mu \mathrm{m}$, and (c) $50 \mu \mathrm{m}$.

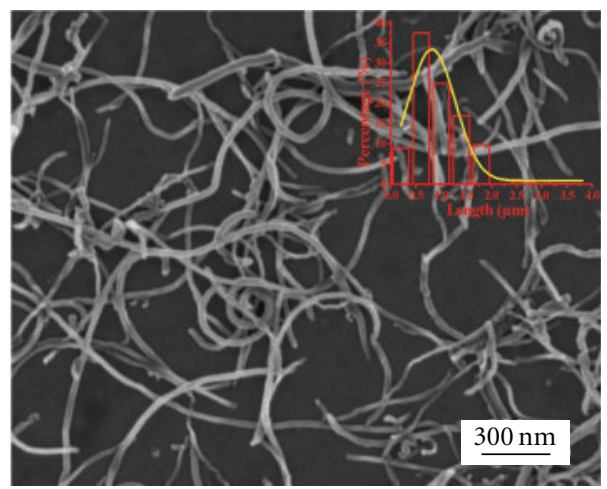

(a)

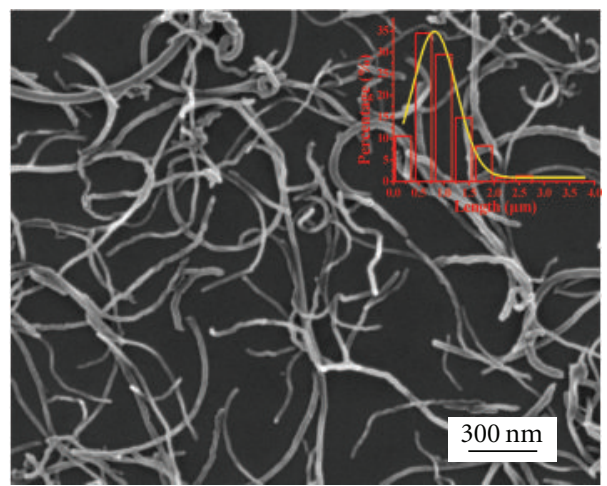

(c)

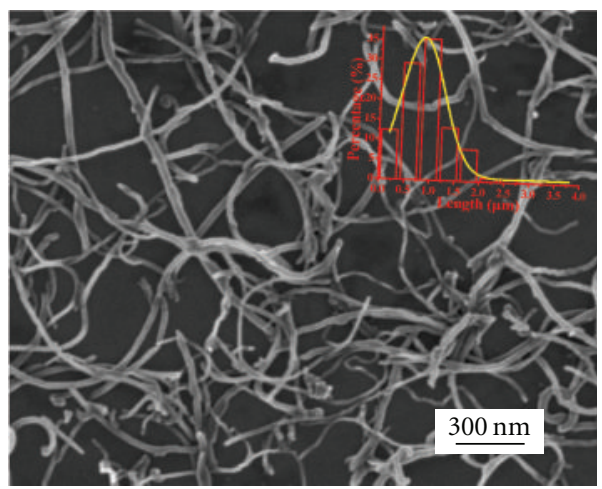

(b)

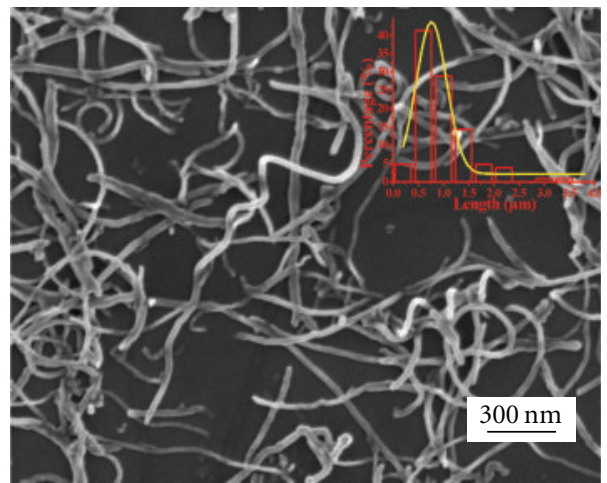

(d)

FIgURE 2: SEM images for oxidized l-MWCNTs: (a) 1-MWCNT-O ${ }_{1}$, (b) 1-MWCNT-O ${ }_{2}$, (c) 1-MWCNT-O , $_{3}$ and (d) 1-MWCNT-O . $^{-}$

of culture medium $(-17 \mathrm{mV}$ as determined). This indicated that the four kinds of MWCNTs absorbed serum protein molecules onto their surface. And, with the oxidation degree increased, Zeta potential value exhibited a decreasing tendency, which implied that l-MWCNTs with higher oxidation degree had less negative surface charges than those with lower oxidation degree. A possible explanation is that Zeta potential of serum protein could be affected by oxidized 1MWCNTs with a higher oxidation degree.

For those dispersing in water, it could be noticed that the size distribution of the four kinds of oxidized 1MWCNTs became more narrow compared with as-received
l-MWCNTs. Among the four oxidized l-MWCNTs, 1MWCNTs- $\mathrm{O}_{4}$ had the most narrow size distribution (Figure $3(\mathrm{~b})$ ), which is consistent with the observations from SEM. These results implied that oxidation treatment with long-time sonication would benefit size homogeneity of carbon nanotubes. The four kinds of oxidized 1-MWCNTs dispersing in the culture medium showed a tendency of size distribution similar to those dispersing in water (Figure 3(d)). Comparing the results given in Figures 3(a) and 3 (c), the average hydrodynamic size for the four kinds of MWCNTs dispersing in the medium was larger than that dispersing in water, which provided further evidence of 


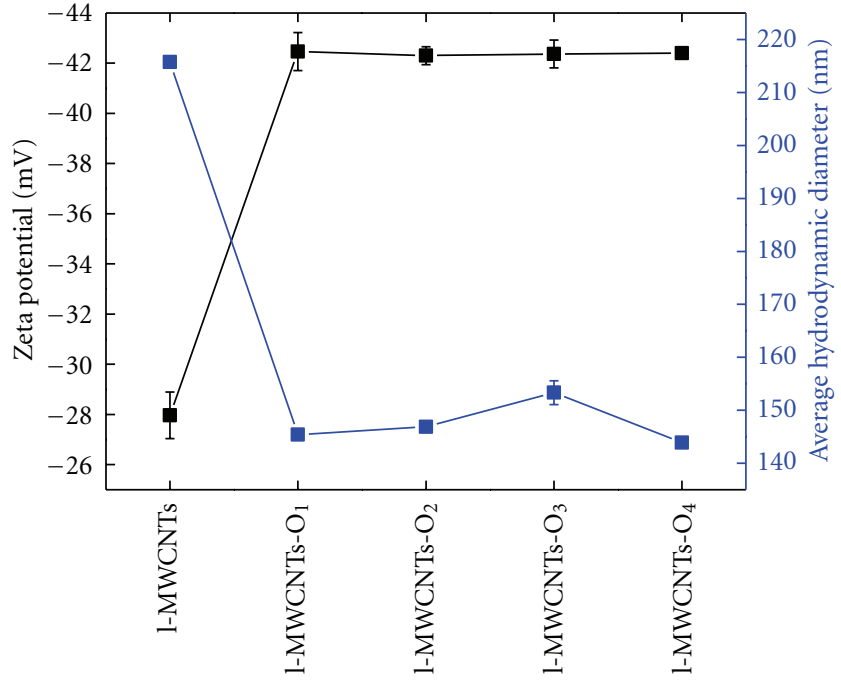

- Zeta potential $(\mathrm{mV})$

$\rightarrow-$ Average hydrodynamic diameter $(\mathrm{nm})$

(a)

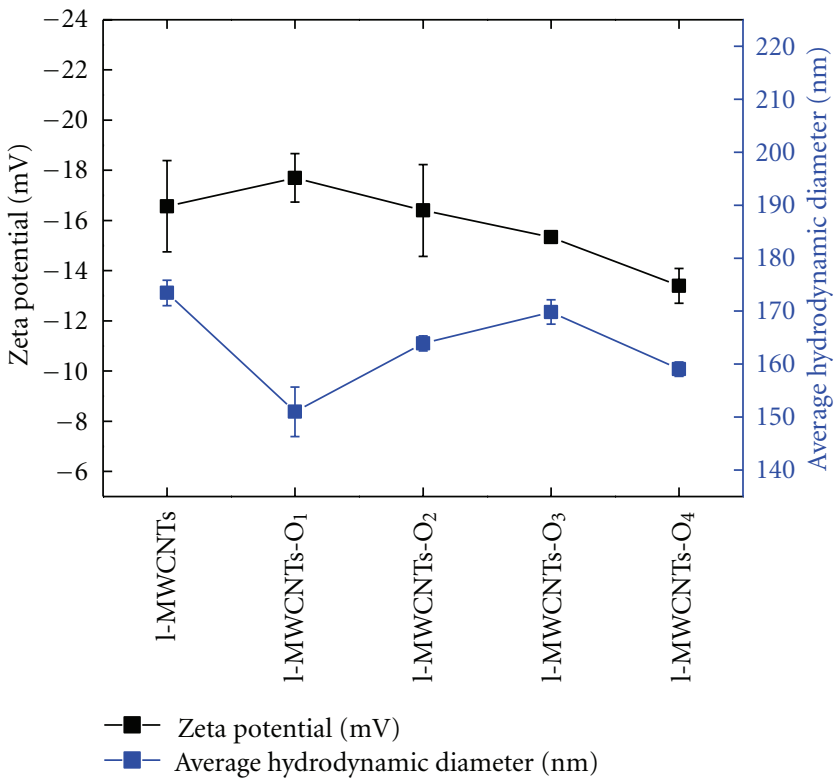

(c)

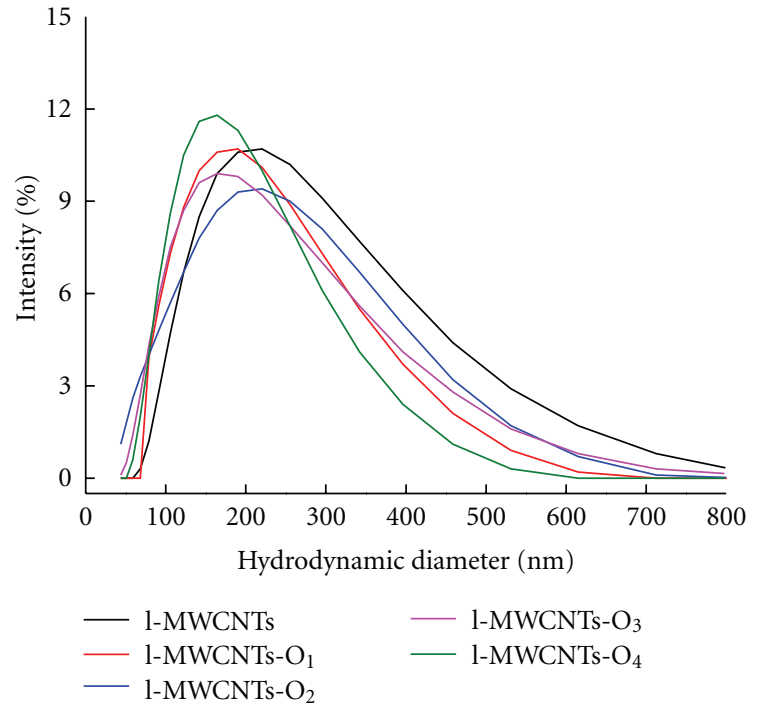

(b)

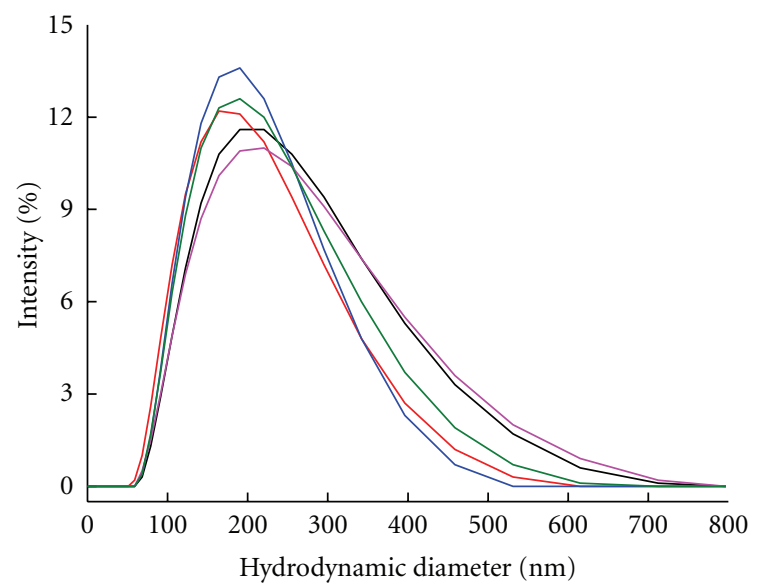

$\begin{array}{ll}- \text { 1-MWCNTs } & \text { 1-MWCNTs-O } \\ \text { 1-MWCNTs-O } & \text { 1-MWCNTs-O } \\ \text { 1-MWCNTs-O } & \text { l-MW }\end{array}$

(d)

FIGURE 3: DLS analysis of as-received l-MWCNTs and the four oxidized l-MWCNTs dispersing in water, in which, (a) and (c) give average hydrodynamic diameters and Zeta potentials of as-received l-MWCNTs and the four kinds of oxidized l-MWCNTs dispersing in water and in the culture medium; (b) and (d) present DLS spectra of the four kinds of oxidized l-MWCNTs dispersing in water and in the culture medium, respectively.

serum protein adsorption. Taken in all, the average hydrodynamic diameter of the four oxidized 1-MWCNTs decreased significantly in reference with as-received l-MWCNTs no matter dispersing in water or in the culture medium, and the four oxidized samples exhibited average size in a closed level.

\subsection{Surface Chemistry of Oxidized MWCNTs (XPS and FTIR).} Water dispersion of carbon nanotube-based materials largely depends on their surface chemistry. Usually treatment of concentrated acids combining with sonication makes carbon nanotubes oxidized, which introduces a variety of oxygencontaining groups to the surface of carbon nanotubes such as $\mathrm{O}-\mathrm{C}=\mathrm{O}, \mathrm{C}=\mathrm{O}$, and $\mathrm{C}-\mathrm{O}$ along with cutting carbon nanotubes short.

As shown in XPS spectra of oxidized l-MWCNTs (Figure $4(\mathrm{a})$ ), the characteristic binding energy of $284.4 \mathrm{eV}$, $285.4 \mathrm{eV}, 286.9 \mathrm{eV}, 288.6 \mathrm{eV}$, and $290.8 \mathrm{eV}$ was attributed to $\mathrm{C}-\mathrm{C}, \mathrm{C}-\mathrm{O}, \mathrm{C}=\mathrm{O}, \mathrm{O}-\mathrm{C}=\mathrm{O}$ and $\pi-\pi^{*}$, respectively. It is 


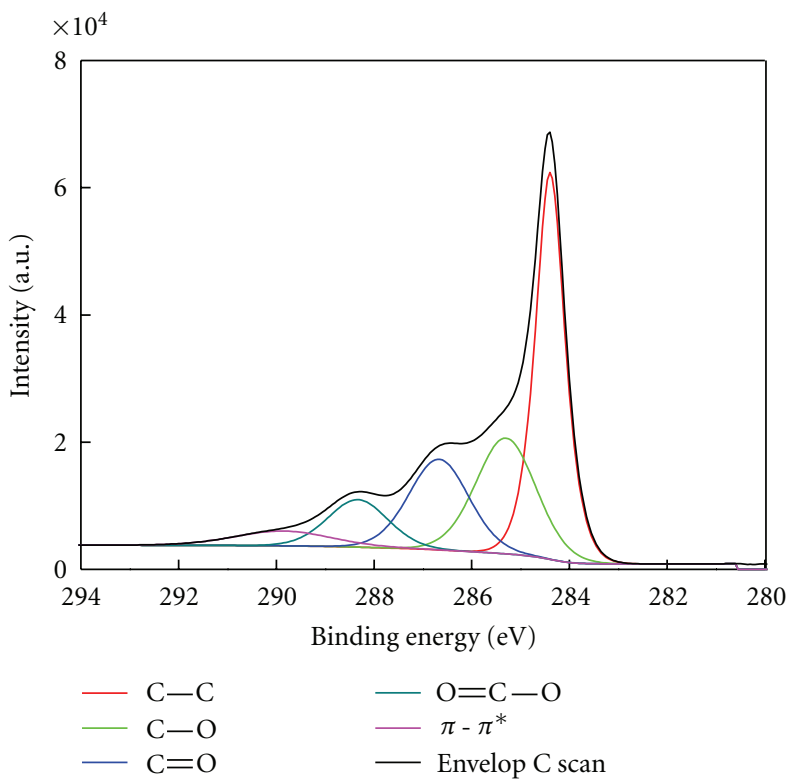

(a)

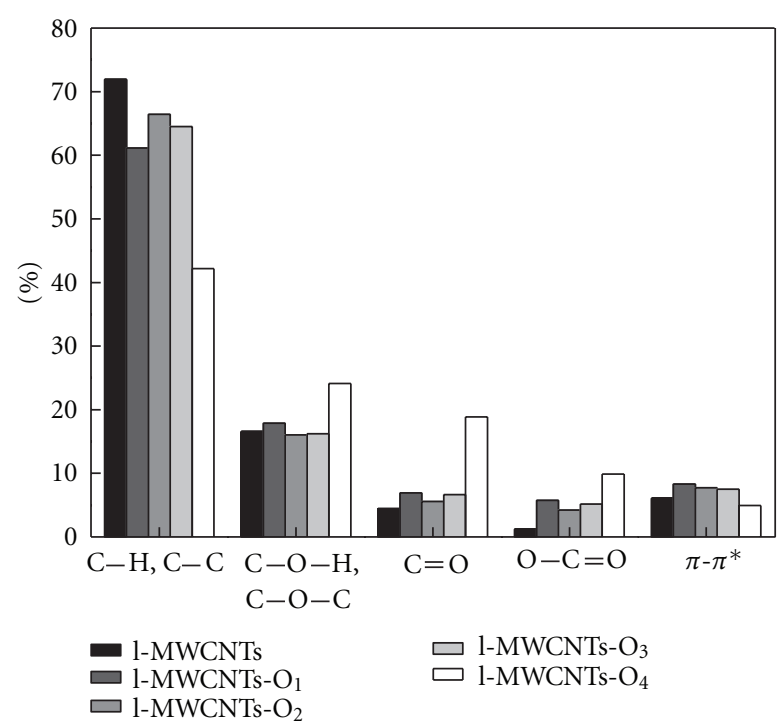

(b)

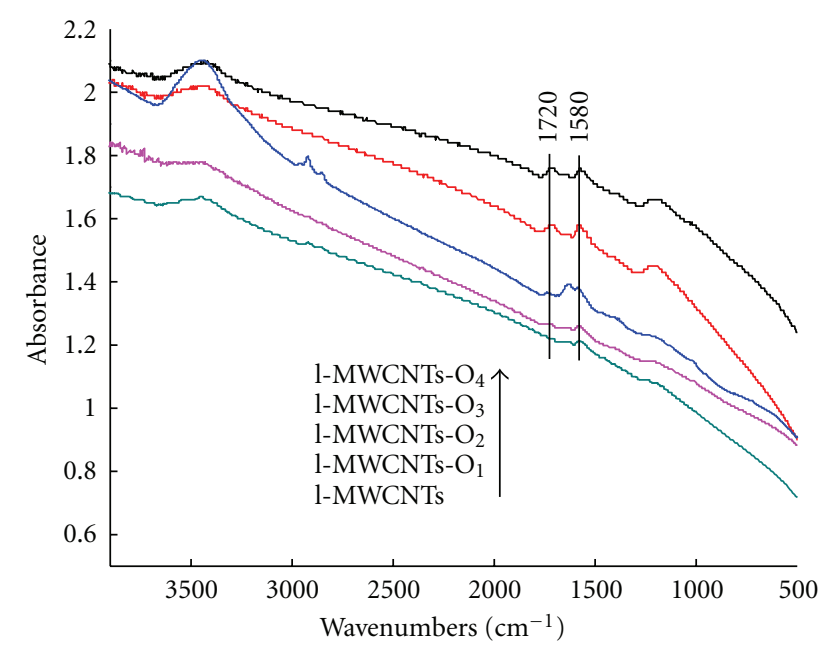

(c)

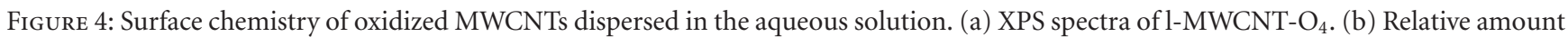
of various oxygen species. (c) FTIR of l-MWCNTs with different oxidation degrees.

important to note that, with the sonication time increased, the amount of oxygen-containing groups increased correspondingly in particular, the order of increasing rate for the different oxygen-containing groups is; $\mathrm{O}-\mathrm{C}=\mathrm{O}>\mathrm{C}=\mathrm{O}$ $>\mathrm{C}-\mathrm{O}$ (Figure 4(b)). FTIR spectroscopy provided further evidence of $\mathrm{O}-\mathrm{C}=\mathrm{O}$ group that existed on the surface, and the characteristic absorption peak of $1720 \mathrm{~cm}^{-1}$ became stronger as sonication time increased (Figure 4(c)). The above results evidenced that longer sonication time increased the oxidation degree of carbon nanotubes, resulting in more oxygen-containing groups on the surface of l-MWCNTs.

3.4. TGA Analysis of As-Received MWCNTs and Oxidized lMWCNTs. TGA can be used to analyze the quality of carbon nanotubes [23] as well as to track the effects of purification process and monitor how changes in manufacturing conditions affect the percentage of carbon nanotubes within the sample [24]. Figure 5 showed TGA and DTG spectra of the three kinds of as-received MWCNTs and the four oxidized l-MWCNTs. The primary oxidation temperature for each material is defined as the temperature at the highest peak for the material on the derivative weight curve and can represent the thermal stability of the material. For the as-received MWCNTs, the oxidation temperatures were $651^{\circ} \mathrm{C}$ for $1-\mathrm{MWCNTs}, 620^{\circ} \mathrm{C}$ for m-MWCNTs, and $610^{\circ} \mathrm{C}$ for s-MWCNTs (Figure 5(a)), among which as-received 1MWCNTs exhibited the highest oxidation temperature. As given by the manufacturer, the oxygen content for the different as-received MWCNTs is $4 \%, 5 \%$, and $6 \%$ for s-, m-, 


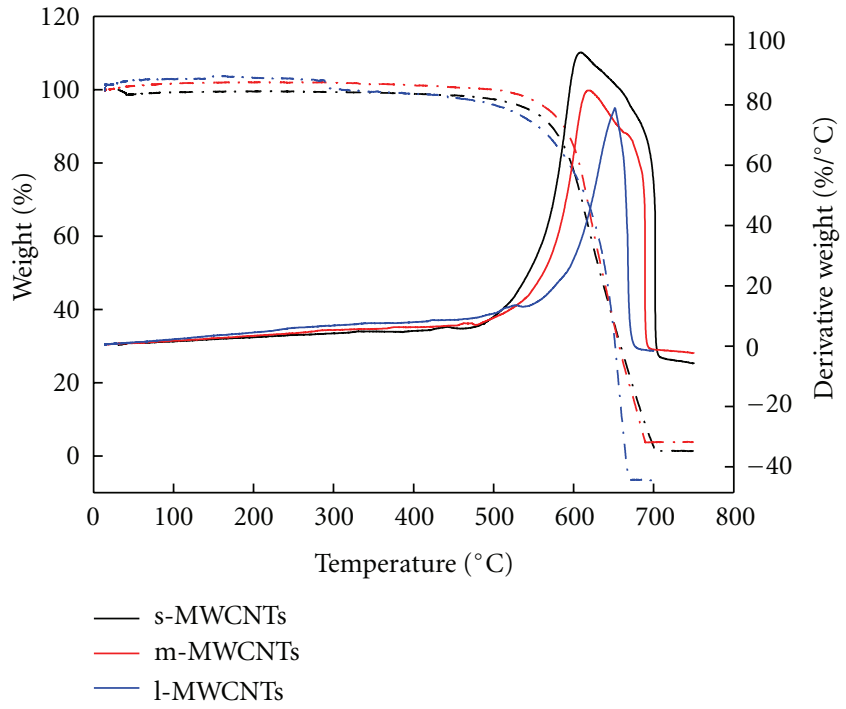

(a)

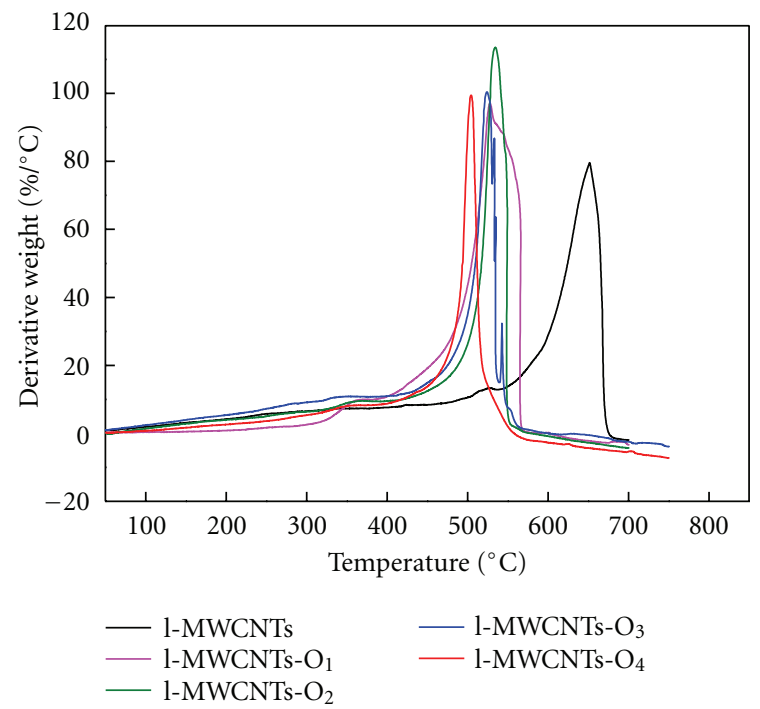

(b)

FIGURE 5: TGA and DTG spectra of as-received s-, m-, l-MWCNTs (a), and oxidized l-MWCNT (b).

and l-MWCNTs, respectively. It was indicated that the surface oxidation degree for the three of as-received MWCNTs was s- >m- >l-MWCTs because lowly oxidized carbon nanotubes are more resistant to decomposition than highly oxidized ones. These are in consistence with the data given by the manufacturer. In addition, s-MWCNTs and m-MWCNTs exhibited a fairly broad decomposition peak with multiple shoulders, which should be likely indicative of multiple types of carbons decomposing. This was consistent with the SEM observations (Figure 1).

Figure 5(b) showed result of TGA tests for the oxidized l-MWCNTs that have similar length distributions. The oxidation temperature of oxidized MWCNTs was $527.83^{\circ} \mathrm{C}$, $534.62^{\circ} \mathrm{C}, 524.31^{\circ} \mathrm{C}$, and $504.4^{\circ} \mathrm{C}$ to l-MWCNT-O ${ }_{1}$, l-
MWCNT-O 2 , l-MWCNT-O 3 , and l-MWCNT-O ${ }_{4}$, respectively. From the temperature data, first, it could be seen that oxidation temperature of the different oxidized 1MWCNTs with aid of sonication was decreased with the increasing oxidation time. It has been reported in the literature that the shift to lower temperature is consistent as the oxygen content increases [23]. Hence, it is inferred that longer sonication time resulted in higher oxidation degree. The results also showed that oxidation temperature of l-MMCNTs- $\mathrm{O}_{1}$ was lower and the peak was broader compared to the other three kinds of oxidized l-MWCNTs, from $410^{\circ} \mathrm{C}$ to $566^{\circ} \mathrm{C}$, indicating that treatment with the concentrated acids only would result in an unhomogeneous oxidization of carbon nanotubes. We would suggest that the part of lowly oxidized nanotubes in l-MMCNTs- $\mathrm{O}_{1}$ made its decomposition temperature increase. Beside the variation of oxidation temperature, the oxidation peaks for the four oxidized 1-MWCNTs were much narrower than the one for as-received 1-MWCNTs, which indicated a sample of higher purity.

3.5. Characteristic Absorption of MWCNTs and Relation to the Size Distribution and Surface Chemistry. MWCNTs showed characteristic absorption spectra in $240 \sim 265 \mathrm{~nm}$. Figures 6(a) and 6(b) presented representative UV spectra of as-received MWCNTs with different average length and the oxidized 1-MWCNTs with different oxidation degrees, respectively, exhibiting the characteristic absorption. It could be seen that the spectra containing multiple peaks, which were resolved into three peaks as shown in Figure 6(c), among them peak 2, was a major one both in intensity and in area. For the three as-received MWCNTs samples, the wavelength of peak 2 shifted towards red obviously from $259 \mathrm{~nm}$ to $262 \mathrm{~nm}$ with the average length of MWCNTs increasing from $2 \mu \mathrm{m}$ to $50 \mu \mathrm{m}$, while the intensity of peak 2 varied from 0.29 to 0.33 (Figure 6(d)). For the four oxidized l-MWCNTs, with the percentage of surface oxygen content increased, the intensity of peak 2 increased correspondingly, from 0.38 to 0.57 (Figure 6(e)), while the wavelength of peak 2 exhibited a slight red shift, from $263 \mathrm{~nm}$ to $264 \mathrm{~nm}$. Together in all, it could be found that the intensity of peak 2 reflected the variation of oxidation degree of the MWCNTs, and the wavelength of peak 2 reflected the majority length of MWCNTs dispersing in water. And, from the resolved spectra, one can identify and compare MWCNTs samples from different sources.

3.6. Colloid Stability of Oxidized MWCNTs. The characteristic absorption of MWCNTs can be used to examine colloid stability of MWCNTs. L-MWCNTs- ${ }_{4}$ was taken as an example in this work; and dits colloid stability was monitored using the absorption spectra. Figure 7 presented the absorption peak of l-MWCNTs- $\mathrm{O}_{4}$ dispersing in water within storage periods. When the absorption spectra were resolved into three peaks, the absorption intensity decreased with time under static condition. The dramatic variation occurred within 17 days, and then the variation extent 

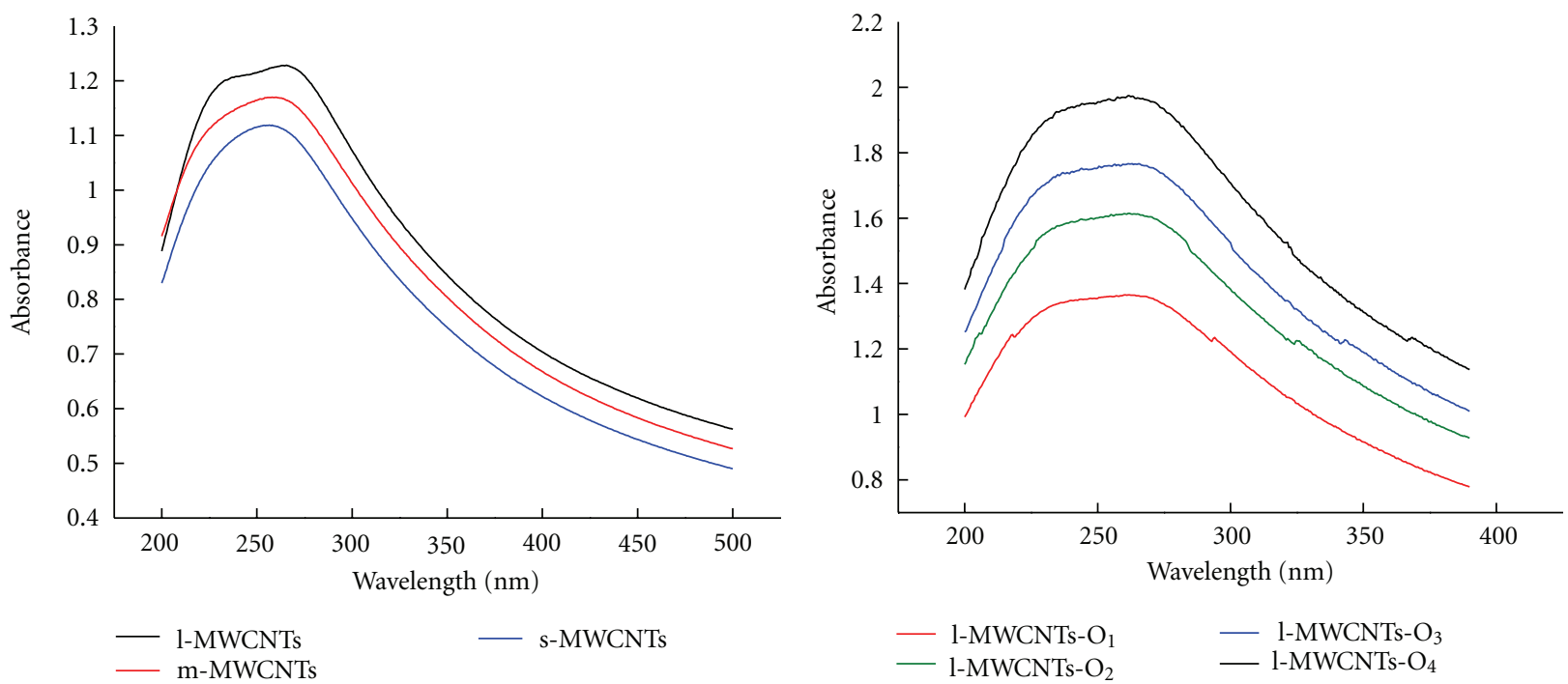

(a)

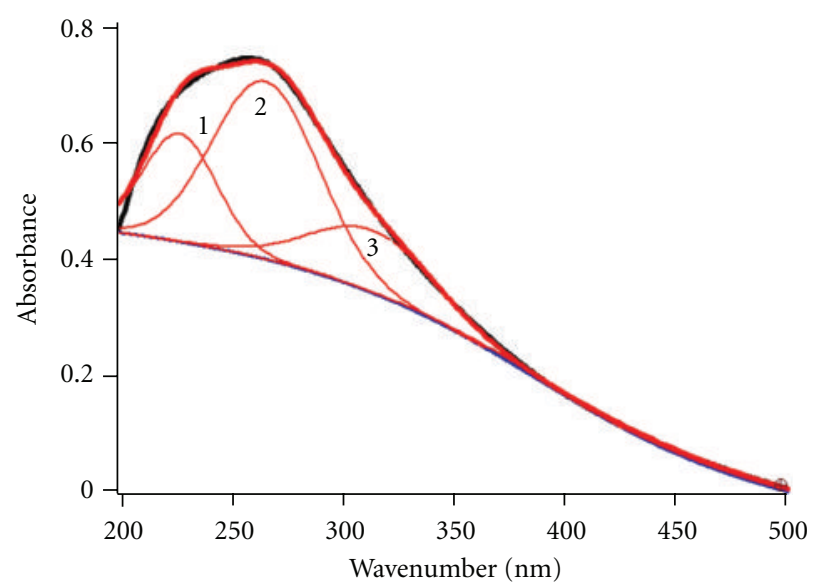

(b)

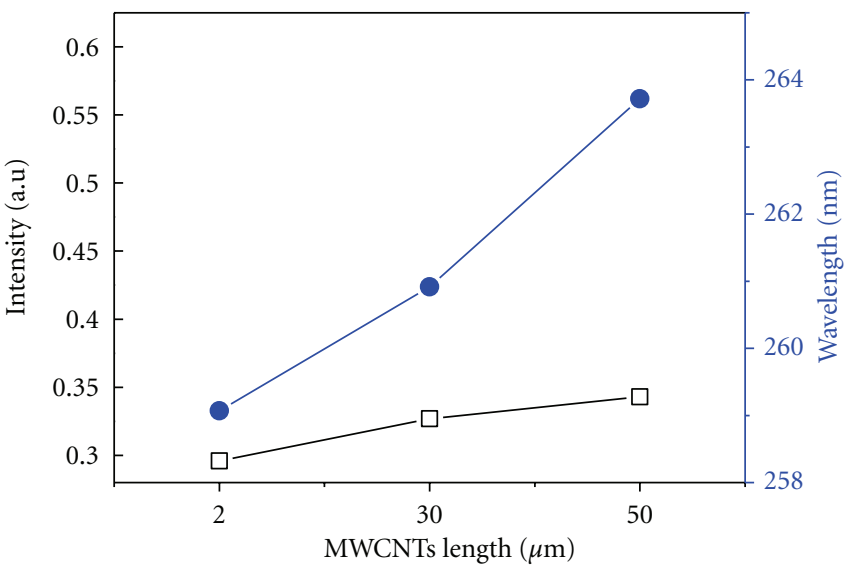

$-\square-$ Intensity

- Wavelength

(c)

(d)

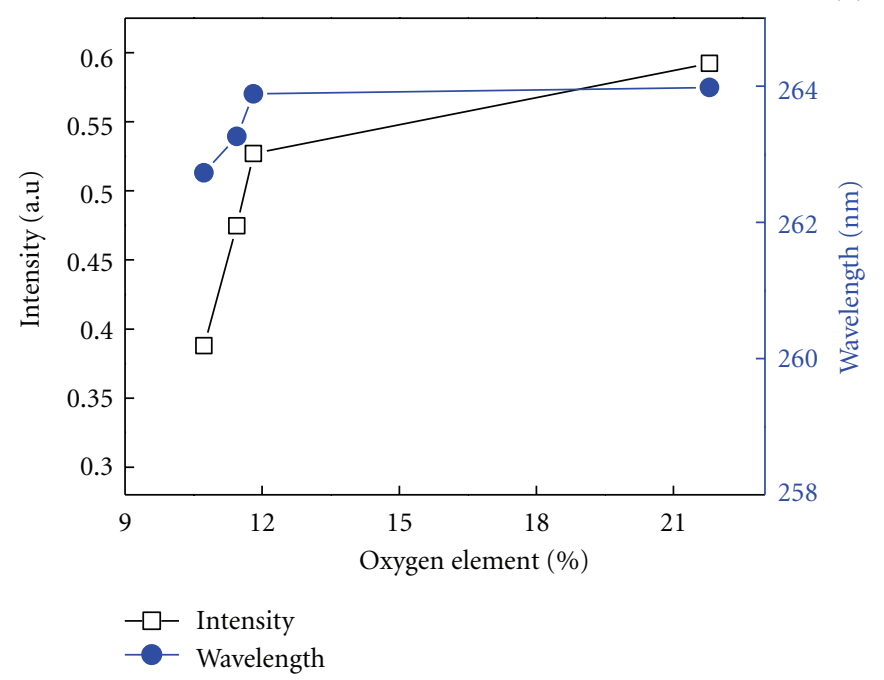

(e)

FIGURE 6: UV spectroscopy of MWCNTs, in which (a) is UV spectra of as-received MWCNTs with different average lengths, (b) is UV spectra of the four kinds of oxidized l-MWCNTs, (c) presents representative spectra of MWCNTs containing three resolved peaks, and (d) and (e) display the relation of intensity and wavelength of peak 2 with as-received MWCNTs and oxidized MWCNTs, respectively. 


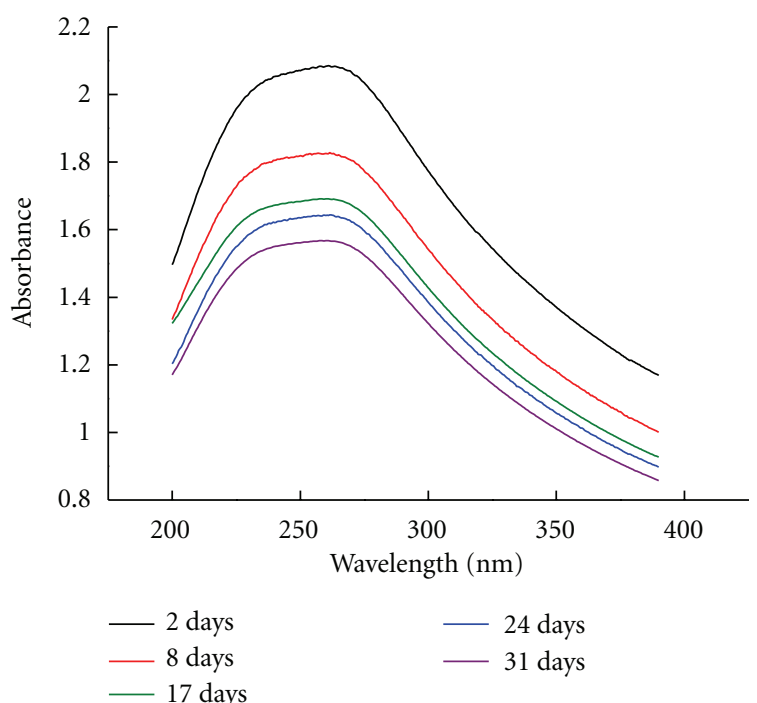

(a)

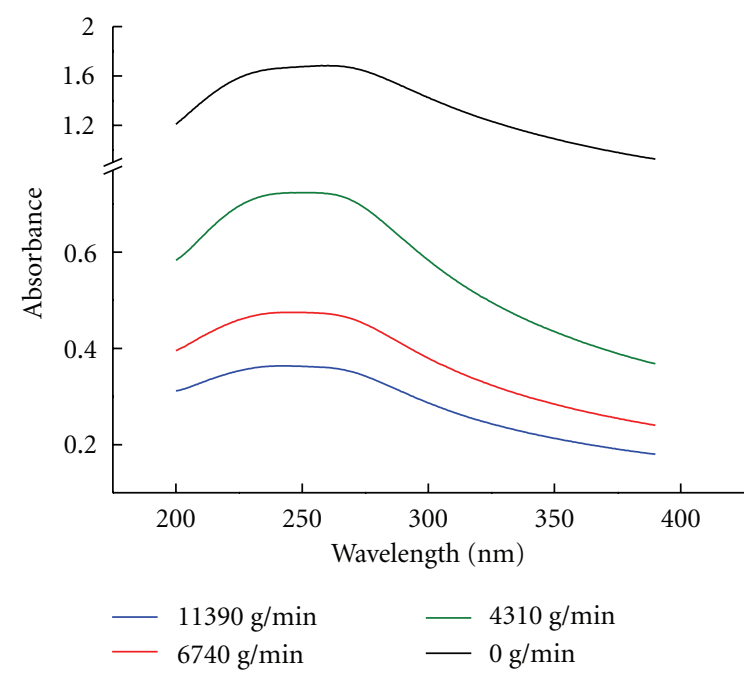

(c)

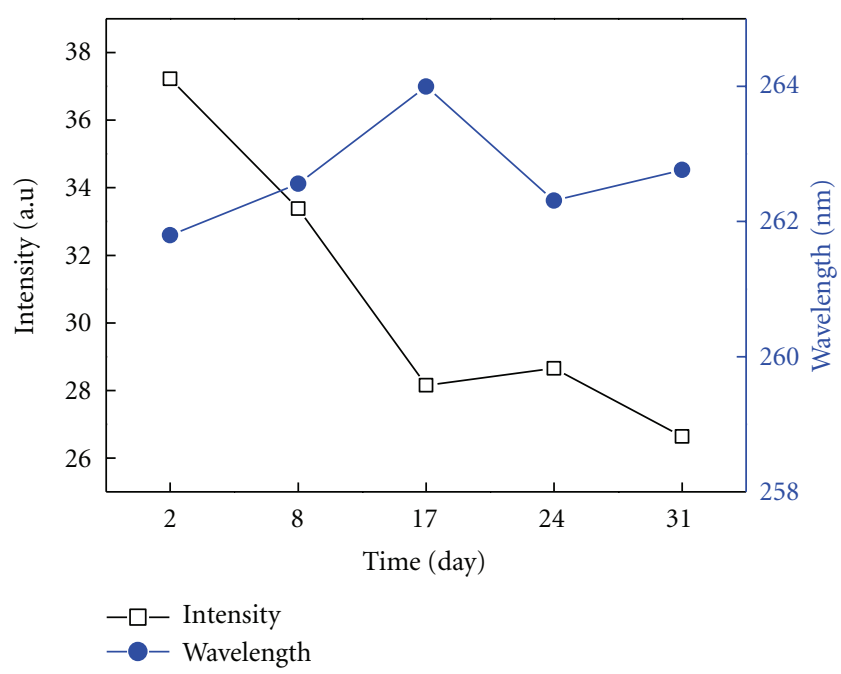

(b)

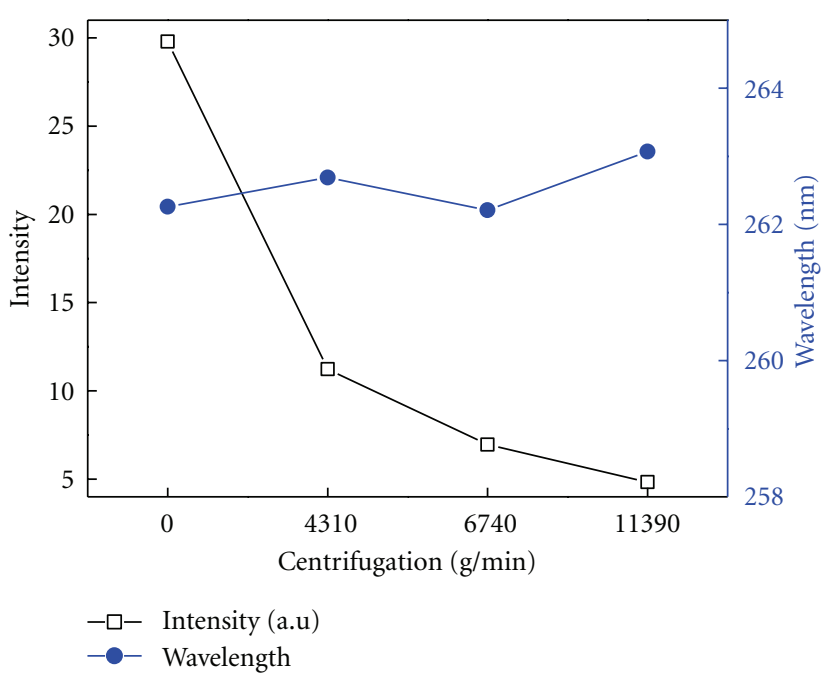

(d)

FIgURE 7: Absorption spectra of l-MWCNTs- $\mathrm{O}_{4}$ under static (a) and centrifugation condition (c); peak 2 wavelength and intensity under static (b) and centrifugation condition (d).

became smaller. Peak 2 also exhibited a slight red shift from 261.7 to 262.3 except the one on day 17, which was $263.9 \mathrm{~nm}$ (Figures 7(a) and 7(b)). The wavelength on day 17 could be attributed to the dispersion status of $1-$ MWCNTs- ${ }_{4}$ in water, the carbon nanotubes dispersing in water were gradually forming agglomerate, which made absorption wavelength red shifted, and then the agglomerate was gradually aggregated and left from water phase within 17 days; the absorption wavelength of the solution then shifted towards back. This is consistent with the variation of absorption intensity.

When centrifugation was applied to the solutions of l-MWCNTs- $\mathrm{O}_{4}$ stored for different time, the wavelength and intensity of peak 2 decreased significantly, while the peak wavelength changed little (Figures $7(\mathrm{c})$ and $7(\mathrm{~d})$ ).
This implied that the length distribution of the carbon nanotubes staying in the water phase after centrifugation was similar, which can be explained by that some highly dispersing carbon nanotubes would come to a relative stability by centrifugation. And it is also suggested that proper centrifugation may speedup the process of obtaining a relative stable colloid solution of MWCNTs.

3.7. Influence of Oxidized l-MWCNTs on Endothelial Proliferation. The proliferation of endothelial incubated with different oxidized 1-MWCNTs was showed in Figure 8. At the low concentration of $0.01 \mathrm{mg} / \mathrm{mL}$, it could be seen that the different oxidized 1-MWCNTs resulted in slight reduction of cell viability than that of control after $48 \mathrm{~h}$ 


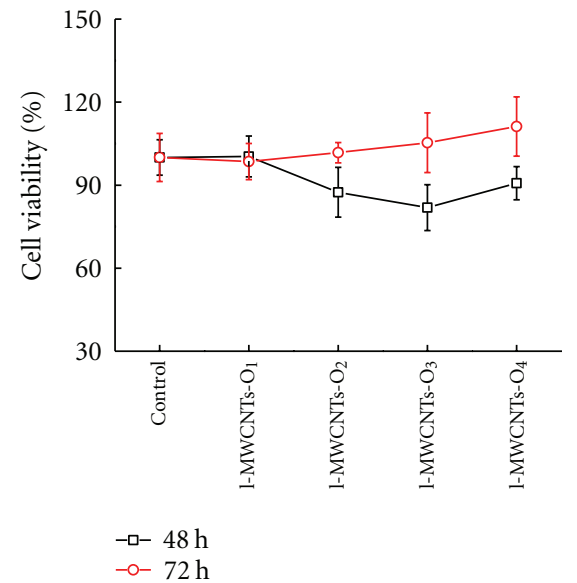

(a)

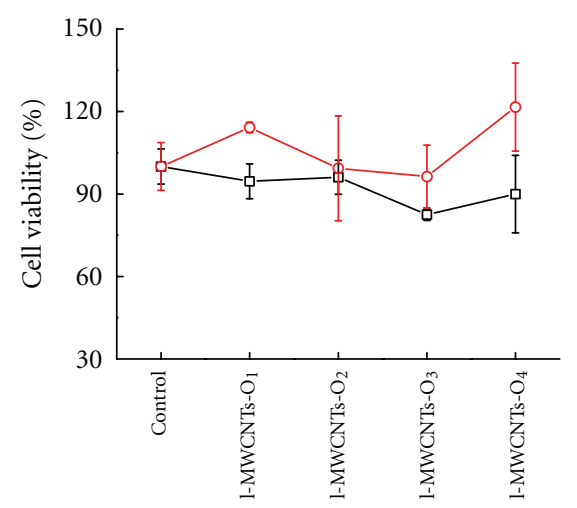

$-\square-48 \mathrm{~h}$

$-0-72 \mathrm{~h}$

(d)

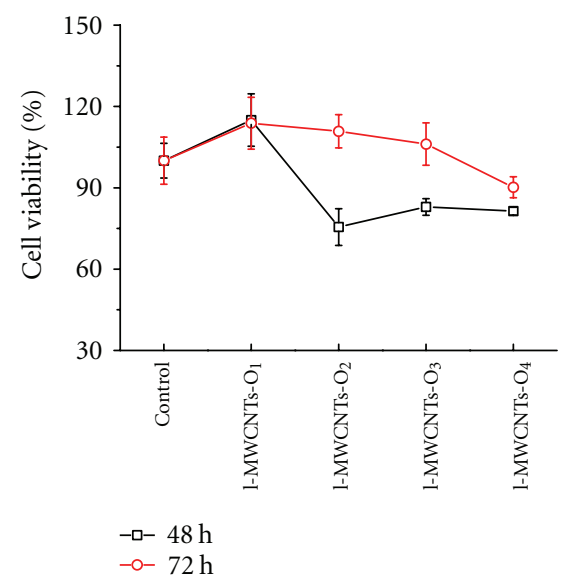

(g)

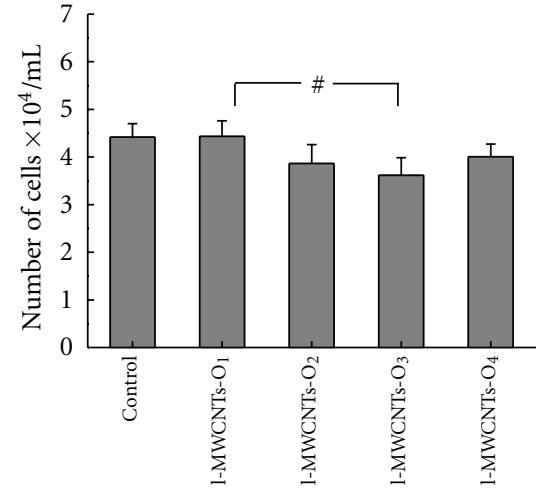

(b)

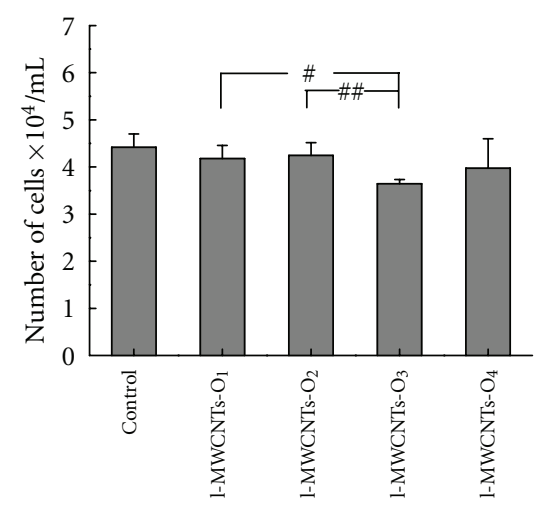

(e)

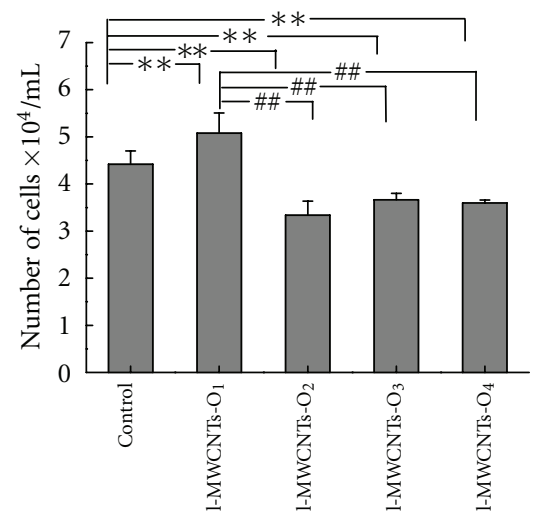

(h)

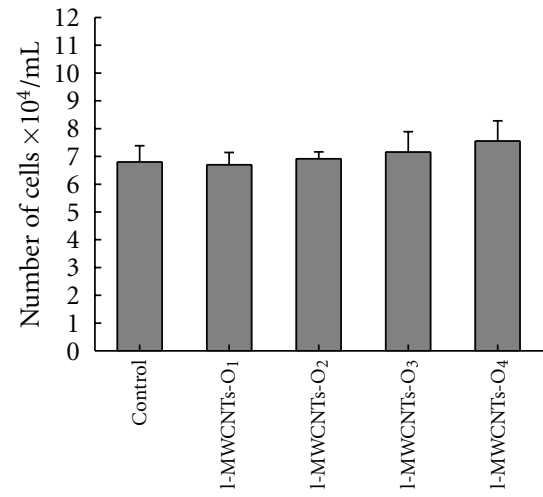

(c)

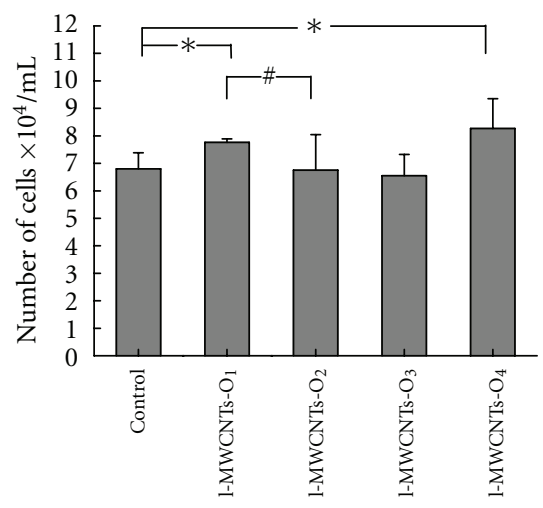

(f)

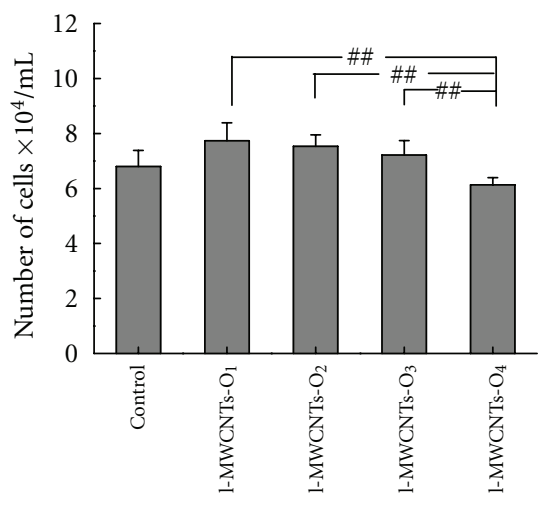

(i)

FIGURE 8: Cell proliferation of the endothelium cells cultivated different concentrations of oxidized l-MWCNTs. (a)-(c) 0.01 mg/mL; (d)-(f) $0.05 \mathrm{mg} / \mathrm{mL}$; (g)-(i) $0.25 \mathrm{mg} / \mathrm{mL}$. The cultivation time for (b), (e), and (h) is $48 \mathrm{~h}$ and for (c), (f), and (i) is $72 \mathrm{~h}$.

of cultivation; however, there was no significant difference between the different oxidized MWCNTs and control. Significant difference appeared in $1-\mathrm{MWCNT}_{3} \mathrm{O}_{3}$ and $\mathrm{l}-$ MWCNT- $\mathrm{O}_{1}$. After $72 \mathrm{~h}$ of cultivation, the cell viability of each group became normal (Figures $8(\mathrm{a})-8(\mathrm{c})$ ). At the middle concentration of $0.05 \mathrm{mg} / \mathrm{mL}$, there was a similar tendency to that at $0.01 \mathrm{mg} / \mathrm{ml}$ after $48 \mathrm{~h}$ of cultivation. Significant difference appeared between l-MWCNT- $\mathrm{O}_{1}$ and 
l-MWCNT-O ${ }_{3}$, and l-MWCNT- $\mathrm{O}_{2}$ and 1-MWCNT-O $\mathrm{O}_{3}$ at $48 \mathrm{~h}$ of cultivation. As the time was increased to $72 \mathrm{~h}$, cell viability also showed a recovery to normal, and l-MWCNTs$\mathrm{O}_{1}$ and 1-MWCNTs- $\mathrm{O}_{4}$ exhibited stimulation to the cells proliferation (Figures $8(\mathrm{~d})-8(\mathrm{f})$ ). Significant difference of proliferation inhibition existed between $1-\mathrm{MWCNT}^{-\mathrm{O}_{1}}$ and l-MWCNT- ${ }_{3}$. At the high concentration of $0.25 \mathrm{mg} / \mathrm{mL}$ (Figures 8(g)-8(i)), profile for the cells cultivated with the four oxidized l-MWCNTs for $48 \mathrm{~h}$ was obviously different from those cultivated at the low or middle concentration; the four different oxidized l-MWCNTs displayed significant inhibition to the cells proliferation in reference to control. Treatment of $0.25 \mathrm{mg} / \mathrm{mL}$ l-MWCNTs- $\mathrm{O}_{2}$ resulted in a $32 \%$ decrease in cell metabolism which is the highest in the current research. After $72 \mathrm{~h}$, cells viability was little different from that of control. It was noticed that there was significant difference between l-MWCNT- $\mathrm{O}_{1}$ and the other three kinds of oxidized MWCNTs at $48 \mathrm{~h}$, while significant difference existed between 1-MWCNT- $\mathrm{O}_{4}$ and the other three kinds of oxidized MWCNTs at $72 \mathrm{~h}$. Taking the above results together, the oxidized l-MWCNTs with different oxidation degree induced different inhibitory effects on the cells proliferation though the variation extents in the groups were not so many. This implied that using the above combined characterization solution might be able to probe the linkage between cells proliferation and different MWCNTs with slight difference. It is suggested that the four oxidized 1-MWCNTs induced different inhibitory effects on the endothelial proliferation; the extent is related to the oxidation degree as well as to the concentration.

In summary, we would suggest that absorption spectroscopy in combination with SEM, DLS, TGA, XPS, and FTIR can provide more characteristic information of length distribution, surface chemistry, and colloid stability and dispersion status for identifying and comparing the dispersion status of multiwalled carbon nanotubes in water. And the obtained detailed information is helpful to compare the biological effects of carbon nanotubes from different sources.

\section{Acknowledgments}

The authors are thankful for financial support from National Key Program of China (973 program 2011CB933504 and 2010CB934002) and Beijing Municipal Natural Science Foundation (2011022). They also thank Dr. Liping Zhang (National Center of Nanoscience and Technology) and Ms. Chaoying Wang (Institute of Physics, CAS) for their kind help in UV-vis-NIR spectroscopy and scanning electron microscopy measurement, respectively. X. Cheng and J. Zhong contributed equally to this work.

\section{References}

[1] S. Beg, M. Rizwan, A. M. Sheikh, M. S. Hasnain, K. Anwer, and K. Kohli, "Advancement in carbon nanotubes: basics, biomedical applications and toxicity," Journal of Pharmacy and Pharmacology, vol. 63, no. 2, pp. 141-163, 2011.
[2] I. Posadas, F. J. Guerra, and V. Ceña, "Nonviral vectors for the delivery of small interfering RNAs to the CNS," Nanomedicine, vol. 5, no. 8, pp. 1219-1236, 2010.

[3] B. Kateb, M. Van Handel, L. Zhang, M. J. Bronikowski, H. Manohara, and B. Badie, "Internalization of MWCNTs by microglia: possible application in immunotherapy of brain tumors," NeuroImage, vol. 37, supplement 1, pp. S9-S17, 2007.

[4] C. Klumpp, K. Kostarelos, M. Prato, and A. Bianco, "Functionalized carbon nanotubes as emerging nanovectors for the delivery of therapeutics," Biochimica et Biophysica Acta, vol. 1758, no. 3, pp. 404-412, 2006.

[5] N. G. Sahoo, H. Bao, Y. Pan et al., "Functionalized carbon nanomaterials as nanocarriers for loading and delivery of a poorly water-soluble anticancer drug: a comparative study," Chemical Communications, vol. 47, no. 18, pp. 5235-5237, 2011.

[6] S. Ilbasmiş-Tamer, Ş. Yilmaz, E. Banoğlu, and I. T. Değim, "Carbon nanotubes to deliver drug molecules," Journal of Biomedical Nanotechnology, vol. 6, no. 1, pp. 20-27, 2010.

[7] N. Kobayashi, M. Naya, M. Ema et al., "Biological response and morphological assessment of individually dispersed multi-wall carbon nanotubes in the lung after intratracheal instillation in rats," Toxicology, vol. 276, no. 3, pp. 143-153, 2010.

[8] A. Takagi, A. Hirose, T. Nishimura et al., "Induction of mesothelioma in $\mathrm{p} 53+/$ - mouse by intraperitoneal application of multi-wall carbon nanotube," Journal of Toxicological Sciences, vol. 33, no. 1, pp. 105-116, 2008.

[9] J. Muller, M. Delos, N. Panin, V. Rabolli, F. Huaux, and D. Lison, "Absence of carcinogenic response to multiwall carbon nanotubes in a 2-year bioassay in the peritoneal cavity of the rat," Toxicological Sciences, vol. 110, no. 2, pp. 442-448, 2009.

[10] Y. Bai, Y. Zhang, J. Zhang et al., "Repeated administrations of carbon nanotubes in male mice cause reversible testis damage without affecting fertility," Nature Nanotechnology, vol. 5, no. 9, pp. 683-689, 2010.

[11] K. Wako, Y. Kotani, A. Hirose, T. Doi, and S. Hamada, "Effects of preparation methods for multi-wall carbon nanotube (MWCNT) suspensions on MWCNT induced rat pulmonary toxicity," Journal of Toxicological Sciences, vol. 35, no. 4, pp. 437-446, 2010.

[12] D. Crouzier, S. Follot, E. Gentilhomme et al., "Carbon nanotubes induce inflammation but decrease the production of reactive oxygen species in lung," Toxicology, vol. 272, no. 13, pp. 39-45, 2010.

[13] Y. Sato, A. Yokoyama, K. I. Shibata et al., "Influence of length on cytotoxicity of multi-walled carbon nanotubes against human acute monocytic leukemia cell line THP-1 in vitro and subcutaneous tissue of rats in vivo," Molecular BioSystems, vol. 1, no. 2, pp. 176-182, 2005.

[14] H. Li, T. Zhang, G. Liang, Y. Zhang, and X. Wang, "In vivo evaluation of acute toxicity of water-soluble carbon nanotubes," Toxicological and Environ-mental Chemistry, vol. 93, no. 3, pp. 603-615, 2011.

[15] J. Meng, J. Duan, H. Kong et al., "Carbon nanotubes conjugated to tumor lysate protein enhance the efficacy of an antitumor immunotherapy," Small, vol. 4, no. 9, pp. 13641370, 2008.

[16] C. Yang, J. Mamouni, Y. Tang, and L. Yang, "Antimicrobial activity of single-walled carbon nanotubes: length effect," Langmuir, vol. 26, no. 20, pp. 16013-16019, 2010.

[17] W. Ma, L. Song, R. Yang et al., "Directly synthesized strong, highly conducting, transparent single-walled carbon nanotube films," Nano Letters, vol. 7, no. 8, pp. 2307-2311, 2007. 
[18] L. Song, L. Ci, L. Lv et al., "Direct synthesis of a macroscale single-walled carbon nanotube non-woven material," Advanced Materials, vol. 16, no. 17, pp. 1529-1534, 2004.

[19] A. Schierz and H. Zänker, "Aqueous suspensions of carbon nanotubes: surface oxidation, colloidal stability and uranium sorption," Environmental Pollution, vol. 157, no. 4, pp. 1088 1094, 2009.

[20] Y. Wang, Z. Iqbal, and S. Mitra, "Rapidly functionalized, water-dispersed carbon nanotubes at high concentration," Journal of the American Chemical Society, vol. 128, no. 1, pp. 95-99, 2006.

[21] Y. K. Moon, J. Lee, J. K. Lee, T. K. Kim, and S. H. Kim, "Synthesis of length-controlled aerosol carbon nanotubes and their dispersion stability in aqueous solution," Langmuir, vol. 25, no. 3, pp. 1739-1743, 2009.

[22] B. Smith, K. Wepasnick, K. E. Schrote et al., "Colloidal properties of aqueous suspensions of acid-treated, multi-walled carbon nanotubes," Environmental Science and Technology, vol. 43, no. 3, pp. 819-825, 2009.

[23] E. Mansfield, A. Kar, and S. A. Hooker, "Applications of TGA in quality control of SWCNTs," Analytical and Bioanalytical Chemistry, vol. 396, no. 3, pp. 1071-1077, 2010.

[24] A. C. Dillon, T. Gennett, K. M. Jones, J. L. Alleman, P. A. Parilla, and M. J. Heben, "Simple and complete purification of single-walled carbon nanotube materials," Advanced Materials, vol. 11, no. 16, pp. 1354-1358, 1999. 

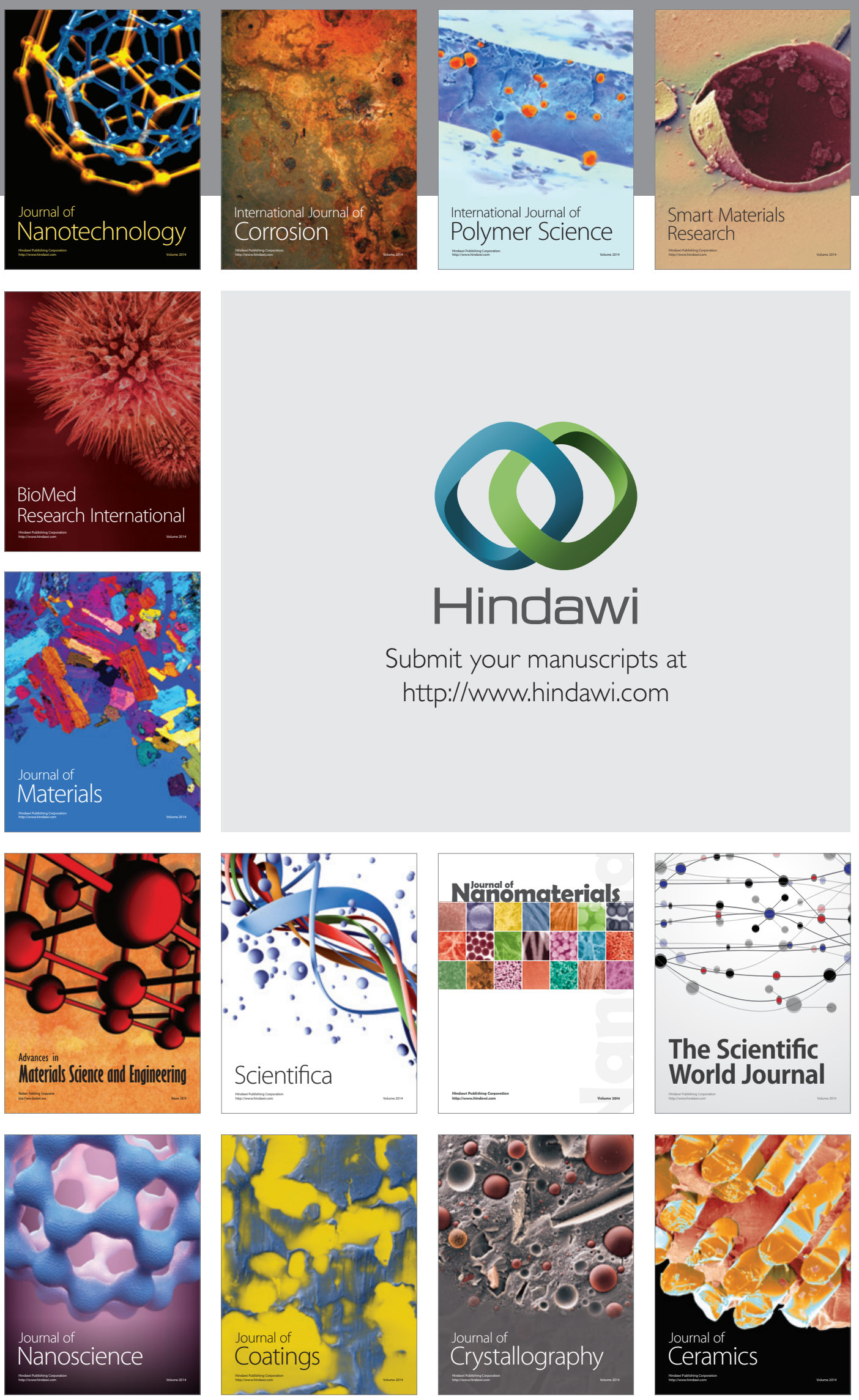

The Scientific World Journal

Submit your manuscripts at

http://www.hindawi.com

\section{World Journal}

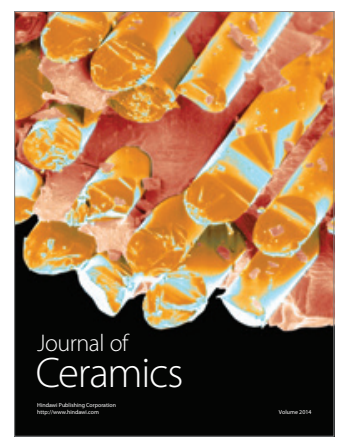

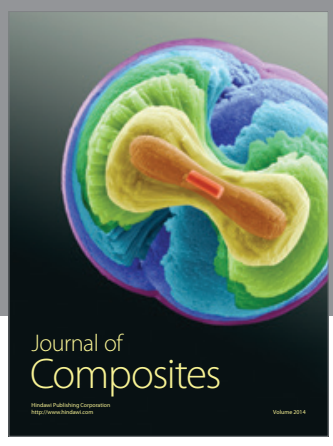
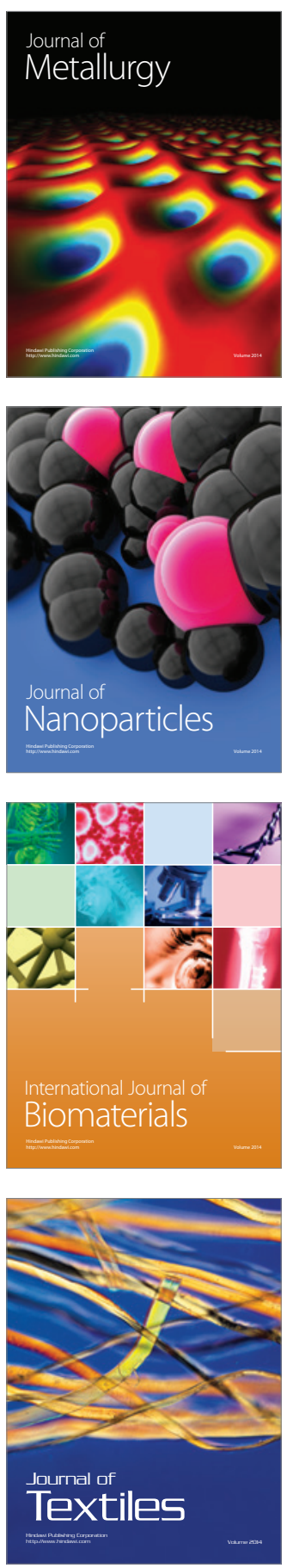